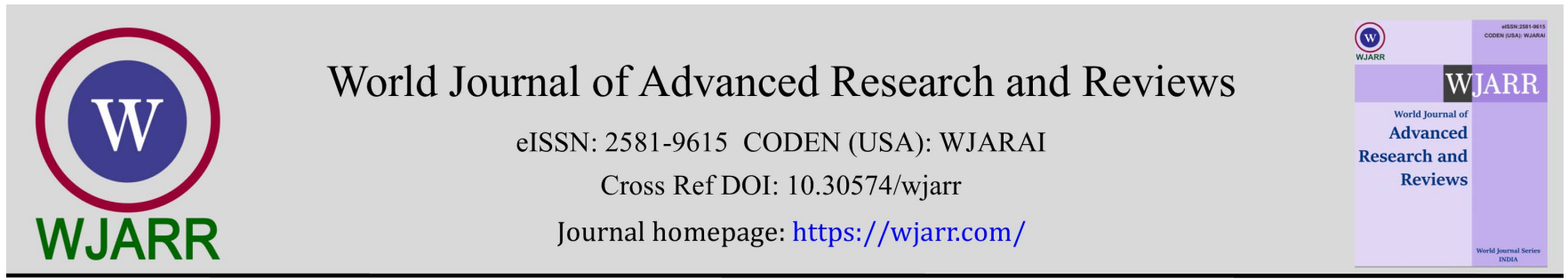

(RESEARCH ARTiCle)

Check for updates

\title{
Effect of mental imagery on depression, anxiety and stress in instituitionalised leprosy patients - An experimental study
}

\author{
Alisha-Akbar-Dossa ${ }^{1, *}$, Parag-Shrinivas-Ranade ${ }^{2}$ and Rahul-Nagendrasingh-Bisen ${ }^{3}$ \\ ${ }^{1}$ Post graduate student, Smt. Kashibai Navale College of Physiotherapy, Narhe, Pune - 41, Maharashtra, India. \\ 2 Professor and Principal, Smt. Kashibai Navale College of Physiotherapy, Narhe, Pune - 41, Maharashtra, India. \\ ${ }^{3}$ Associate Professor, Department Neurophysiotherapy, Smt. Kashibai Navale College of Physiotherapy, Narhe, Pune - 41, \\ Maharashtra, India.
}

World Journal of Advanced Research and Reviews, 2021, 12(03), 296-303

Publication history: Received on 11 November 2021; revised on 13 December 2021; accepted on 15 December 2021

Article DOI: https://doi.org/10.30574/wjarr.2021.12.3.0701

\begin{abstract}
Leprosy, also known as Hansen's disease is caused by Mycobacterium Leprae. Despite being curable, it continues to be a significant health problem in many parts of the world. The prevalence of psychiatric disorders is higher in the leprosy affected population than in the general population. Physical activity has been associated with reduced symptoms of depression and anxiety. It is also associated with improved life satisfaction and psychological well-being. Mental Imagery holds belief as an intervention in the treatment of psychological disorders. This is attributable to its harmless, time and cost effective nature. The study aimed to assess the effect of mental imagery on Depression, Anxiety and Stress in institutionalised Leprosy patients using the DASS-21 (Depression, Anxiety and Stress Scale -21). It was carried out in 34 subjects divided equally in the control and experimental groups. The control group received an aerobic exercise program whereas the experimental group received an aerobic exercise program with mental imagery. It was thus concluded that a statistically significant difference was obtained in the scores of Depression in the experimental group. However, only clinically significant differences could be obtained in the scores of Anxiety and Stress. Thus, mental imagery can be used as an effective adjunct with conventional aerobic exercises for reducing Depression, Anxiety and Stress in institutionalised Leprosy patients.
\end{abstract}

Keywords: Leprosy; Mental Imagery; Aerobic exercises; Depression; Anxiety; Stress

\section{Introduction}

Leprosy is a chronic, infectious disease caused by Mycobacterium Leprae which has an affinity for skin and nerves [1]. The nerve infection produces axonal dysfunction and demyelination which leads to sensory loss and its sequelae of incapacity and deformity [2]. According to the WHO, the prevalence of leprosy corresponds to 22.9 per million population [3]. India achieved the elimination figure of less than 1 case of leprosy per 10,000 people during the month of December, 2005. Despite this, India is still accountable for the largest number of new leprosy cases in the world, contributing to more than fifty per cent of the world leprosy burden [4]. Among the millions of people who have been cured with MDT (Multi drug therapy), there are a large number of people who still suffer from the long-term complications of the disease which include temporary and permanent disability, deformity and social stigma [2]. The prevalence of psychiatric diseases in the Leprosy affected population is higher (99/1000) compared to the general population (63/1000) [5]. Depression is the most common disturbance found amidst these patients. [5,6,7] Shale [8] in her study on women with Hansen's, showed that anxiety disorders were more commonly found in people with leprosy. There is a lack of literature from our country in this important health problem [9]. A multi-centric study carried out by Govindasvamy [10] indicates that more than $30 \%$ of people affected by leprosy have mental health issues, which

\footnotetext{
* Corresponding author: Alisha Akbar Dossa

Post graduate student, Smt. Kashibai Navale College of Physiotherapy, Narhe, Pune - 41, Maharashtra, India.
} 
emphasizes the importance of mental health care services in leprosy. The factors that predispose these patients to developing depression or anxiety are gender (for example, women are at more risk than men), lower levels of education, lower socio-economic status and those with any level of disability. Aerobic Exercise favorably compares to antidepressant medications as a first-line of treatment for mild to moderate depression and has also shown to improve depressive symptoms when used along with medications. It has also been persistently shown to be associated with improved physical being, life satisfaction, mental functioning and psychological well-being [11]. Mental Imagery (MI) can be referred to as the representations and the accompanying experience of sensory information without an actual external stimulus [12]. It plays a pivotal role in many mental disorders and clinicians can utilize it in the treatment of such disorders. This is attributable to its harmless, time and cost effective nature which creates a healing partnership between the professional and the patient [13]. Also there is good patient adherence and no extra physical strain associated with it. It can also be performed with ease in any particular setting. Thus, the study aims to assess the effect of mental imagery on Depression, Anxiety and Stress in institutionalised Leprosy patients.

\section{Aim}

The study aimed to assess the effect of mental imagery on Depression, Anxiety and Stress in institutionalised Leprosy patients using the DASS-21 (Depression, Anxiety and Stress Scale -21).

\section{Material and methods}

A total of 34 subjects residing in an instituitionalised set-up in Pune participated in the study. The sample size was calculated based on the prevalence of psychiatric disorders occurring in these patients in India [14]. The technique of sampling was convenient sampling and randomization of the subjects into two groups was done through a computer generated random allocation chart. Leprosy patients in the age group of 18 to 60 years who possessed basic reading skills of a local language were included in the study. This was a pre-requisite as the screening measure and outcome measure utilised in the study were both self-administered and provided in a language suitable to these patients. The Bett's questionnaire was used as a screening tool to assess the imagery abilities of the patients. Only those patients who scored a minimum of 4 points on each item were recruited in the study (scores in the range of 35 to 140) which was the criteria for possessing basic imagery abilities. Those patients who were in the active phase of infection and those who had major cardiovascular risks interfering with the conventional aerobic exercise protocol were excluded from the study. The DASS-21 (Depression, Anxiety and Stress Scale - 21) was administered at the beginning of the intervention and after 2 weeks. It has excellent Cronbach's alpha values for reliability of $0.81,0.89$ and 0.78 for the subscales of depression, anxiety and stress respectively. It is also found to have excellent concurrent and convergent validities [15]. A conventional protocol was designed for the control group. It consisted of an aerobic exercise program as per the NHS guidelines for reducing depression and anxiety in adults [16]. This included :

- A period of Warm up for 5 - 7 minutes consisting of basic limb movements and flexibility exercises involving the main muscle groups of the body (deltoid, biceps, triceps, trunkal muscles, quadriceps, hamstrings, and gastrocnemius).

- This was followed by aerobic activities lasting 15 - 20 minutes consisting of multiple short bouts of moderate to vigorous intensity exercises involving both upper limbs, lower limbs and trunk - for example, cross body movements, stepping, marching etc with intermittent rest periods.

- The session was terminated by a cool down phase lasting 10 minutes involving Jacobsen's Progressive Muscle Relaxation technique (JPMRT).

The experimental group received the conventional protocol with a mental imagery session lasting 10 minutes. The MI session was delivered via an audiotape with a pre-recorded voice. This was done to maintain uniformity in terms of instructions and voice quality. The mental imagery was validated by a clinical psychologist. The treatment was delivered in the form of group session involving 5-7 people at a time, for both the control and experimental groups. Frequency of treatment for both the groups was four times per week for 2 weeks. A follow up through a diary and phone calls was maintained for the rest of the days.

Permission from the ethical committee of the institute was taken. Written informed consents from the participants were obtained. The subjects were screened according to the inclusion and exclusion criteria. Demographic details and previous medical history noted. The Bett's questionnaire was administered and patients who scored between 35 and 140 were only included in the study as the criteria for possessing minimum imagery abilities. The DASS-21 was then administered to the participants at the start and at the end of 2 weeks. Patients were also asked if they practice some other methods of relaxation such as Yoga, meditation etc or are on medications for depression or anxiety to account for 
factors which may confound the study. These patients were not excluded from the study on grounds of ethical considerations.

\section{Results}

The general characteristics of the subjects were analyzed using descriptive statistics. As the data in the study was non normative, non-parametric tests were used. Wilcoxon signed ranked tests were used for within group comparisons and Mann Whitney tests were used for the inter group comparison. In the study, significance was kept at the $95 \%$ confidence interval.

The mean \pm SD of age of cases studied in the control and experimental group was $61.76 \pm 15.45$ years and $61.06 \pm 10.04$ years respectively. The distribution of the mean age of cases studied, did not differ significantly between the two study groups ( $\mathrm{P}$ value > 0.05). 17 subjects were studied in the control group, of which $13(76.4 \%)$ were males and 4 (23.5\%) were females. 17 cases were studied in the experimental Group, of which 9 (52.9\%) were males and 8 (47.05\%) were females.

Table 1 below shows the mean age, standard deviation, standard error of means and distribution of males and females in the control and experimental groups. The figure below shows a graphical representation for the same.

Table 1 Descriptive statistics of subjects in the control and experimental groups

\begin{tabular}{|l|c|c|}
\hline & Control & Experimental \\
\hline Mean age & 61.76 years & 61.06 years \\
\hline Standard Deviation & 15.45 & 10.04 \\
\hline Standard Error of Mean & 3.748 & 2.345 \\
\hline No. of males & 13 & 09 \\
\hline No. of females & 04 & 08 \\
\hline
\end{tabular}

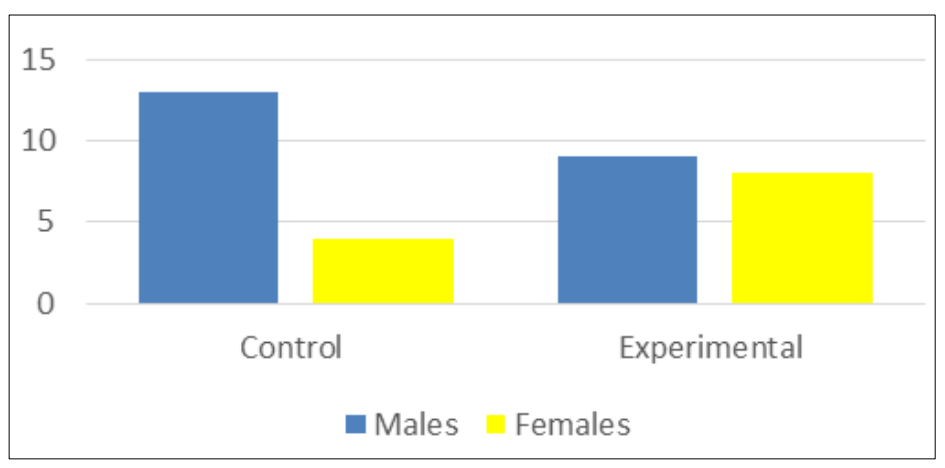

Graph 1 Distribution of males and females in the control and experimental groups

\subsection{Intra group analysis}

\subsubsection{Depression}

A statistically significant difference is obtained in both the conventional and experimental groups. (P value $<0.0001)$.

\subsubsection{Anxiety}

A statistically significant difference is obtained in both the conventional and experimental groups. $(\mathrm{P}$ value $=0.0002)$

\subsubsection{Stress}

A statistically significant difference is obtained in both the conventional and experimental groups. $(\mathrm{P}$ value $<0.0001)$ 


\subsection{Inter group analysis}

\subsubsection{Depression}

A statistically significant difference is obtained between the conventional and experimental groups. ( $p$ value $=0.0037)$

\subsubsection{Anxiety}

A statistically significant difference is not obtained between the conventional and experimental groups. $(\mathrm{P}$ value $=$ 0.3768)

\subsubsection{Stress}

A statistically significant difference is not obtained between the conventional and experimental groups. (P value $>$ 0.9999)

The table and graphs below show the cumulative scores of depression, anxiety and stress in both the control and experimental groups in an intra-group analysis.

Table 2 Intra group analysis of Depression, Anxiety and Stress in the control and experimental groups

\begin{tabular}{|l|c|c|c|c|c|c|}
\hline & \multicolumn{2}{|l|}{ CONTROL (Mean +/- SD) } & P Value & \multicolumn{2}{l|}{ EXPERIMENTAL (Mean +/-SD) } & P Value \\
\hline & PRE & POST & & PRE & POST & \\
\hline DEPRESSION & $11.06+/-3.816$ & $3.29+/-2.995$ & $<0.0001$ & $14.82+/-4.694$ & $2.94+/-1.600$ & $<0.0001$ \\
\hline ANXIETY & $4.118+/-3.967$ & $0.71+/-1.572$ & 0.0002 & $3.412+/-3.374$ & $0.82+/-1.237$ & 0.0002 \\
\hline STRESS & $8.941+/-4.643$ & $2.24+/-2.635$ & $<0.0001$ & $8.824+/-4.187$ & $2.12+/-1.799$ & $<0.0001$ \\
\hline
\end{tabular}

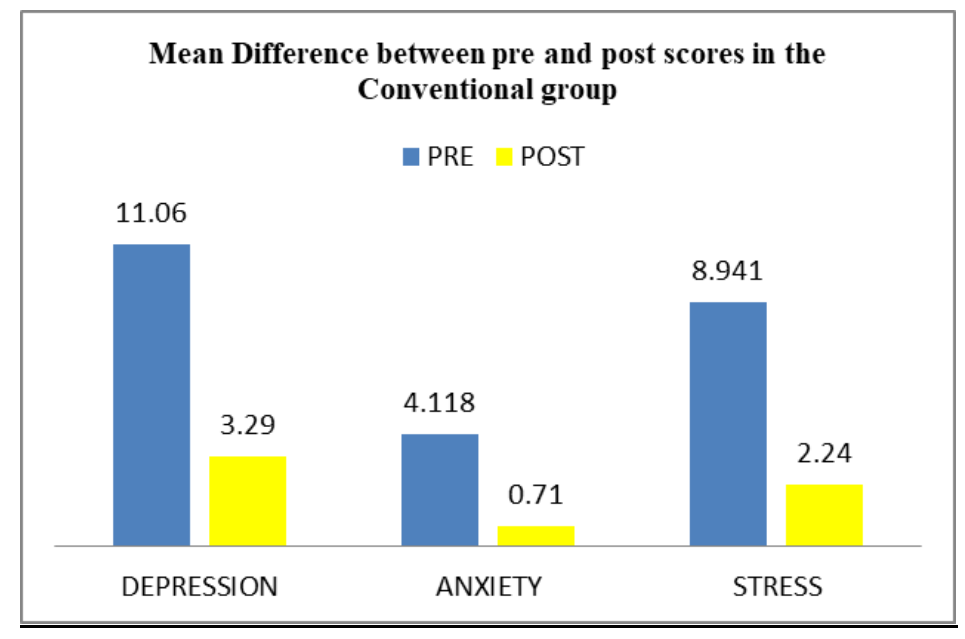

Graph 2 Mean Difference between pre and post scores in the control group 


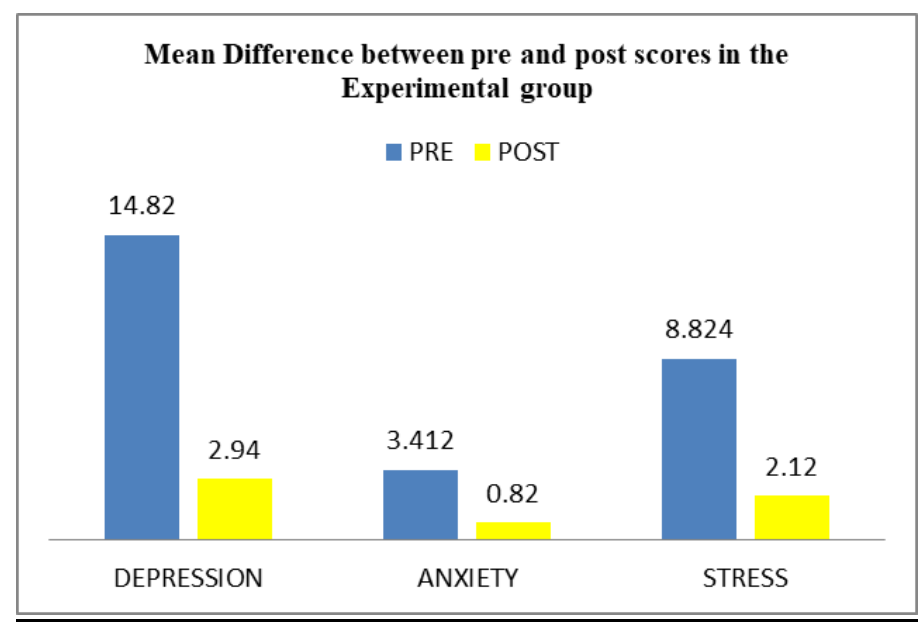

Graph 3 Mean Difference between pre and post scores in the experimental group

The table and the graph below shows the cumulative scores of depression, anxiety and stress in the control and experimental groups in an inter-group analysis.

Table 3 Inter group analysis of Depression, Anxiety and Stress between the control and experimental groups

\begin{tabular}{|l|c|c|c|}
\hline & CONTROL (Mean Diff. +/- SD) & EXPERIMENTAL (Mean Diff. +/- SD) & P Value \\
\hline Depression & $7.765+/-2.905$ & $11.882+/-3.903$ & $\underline{\mathbf{0 . 0 0 3 7}}$ \\
\hline Anxiety & $3.412+/-2.895$ & $2.588+/-2.425$ & 0.3768 \\
\hline Stress & $6.706+/-2.443$ & $6.706+/-3.387$ & $>0.9999$ \\
\hline
\end{tabular}

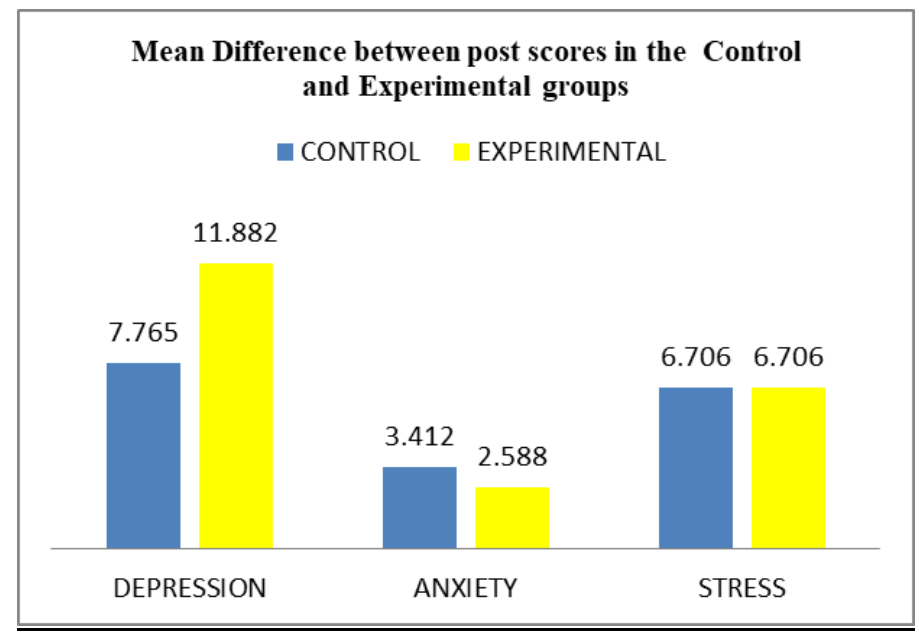

Graph 4 Mean Difference of post scores in the control and experimental groups

\section{Discussion}

A total of 34 participants were recruited and their data was analyzed for descriptive statistics. 22 males and 12 females participated in the study. The mean age of the participants in the control and experimental groups was $61.76+/-15.45$ years and $61.06+/-10.04$ years respectively. None of the patients dropped out of the study.

The data was tested for normalcy and showed non-normative distribution. Hence non-parametric tests were applied for within group and inter-group analysis. Wilcoxon signed ranked tests were used for intra group analysis and Mann Whitney tests were used for inter-group analysis. 
Due to higher mean difference in the scores of depression in the experimental group than control, ( $p$ value $=0.0037)$ it can be concluded that the difference is statistically significant. Victoria and Grace [17] in their report suggest that utilizing emotional mental imagery in psychological interventions could be a promising approach to reduce anxiety and depression. Emotional mental imagery is a powerful part of our mental landscape. It has the capacity to depict, process and generate emotional events and thus it could have an important role in psychological therapies. Mental imagery training can increase the need for rewarding stimuli and reward sensitivity. Higher levels of reward sensitivity and the need for rewarding stimuli, help to identify and motivate repetition of pleasant activities and this behavioral activation, subsequently, is thought to increase positive emotions [17]. Therefore, both the above mentioned mechanisms are highly relevant for providing resilience against depressive symptoms.

Due to nearly similar mean difference values in the experimental and control group for anxiety and stress ( $\mathrm{p}$ value $=$ 0.377 and $>0.999$ respectively), it can be concluded that the difference is not statistically significant between the experimental and control groups for anxiety. This could be attributed to the low baseline scores in these domains at the start of the study. However a clinically significant difference was seen. In a study conducted by Ignacio J et. al [18], there was a significant improvement observed in the performance of nursing students whilst managing their deteriorating patients, using mental imagery. However, no statistical significance was obtained in the pre and post-test heart rate and systolic blood pressure values in the study. A comparison of the State Trait Anxiety Index (STAI) results also did not show significant differences between the pre-post scores. However, a clinical difference was seen. The results obtained in our study are similar to the results of the study described above.

A study conducted by Stephen [19] states that imagery is a cognitive tool that acts as a communication mechanism for the following three characteristics - perception, emotion, and bodily change. Research has stated that imagery can help to decrease pain and stress, change the course of a disease and improve a patient's outlook on their illness. It provides a feeling of sense of control and offers the client an effective tool for self-care. This also provides justification for the improvement seen in the stress scores of the experimental group.

The improvements in the domains of the DASS-21 in the control group can be attributed to a number of mechanisms. Results of cross-sectional and longitudinal studies have more consistently indicated that aerobic exercise training has antidepressant and anxiolytic effects and protects against the harmful consequences of stress. Also, exercise training recruits a process which provides an enduring resilience to stress [20]. The improvements in the scores of depression in the control group can possibly be attributed to the neural mechanisms which mediate the positive effects of exercise on depressive symptoms. Significantly larger effects were found for interventions in major depressive disorder (MDD) which utilized aerobic exercise at moderate and vigorous intensities, both in a supervised and unsupervised format [21]. The prefrontal cortex, anterior cingulate cortex, hippocampus and corpus callosum have emerged as structural neural markers and these may serve as targets for exercise-based treatments for depression [22].

Physical Activity (PA) may act through a range of different physiological and psychological mechanisms for reducing anxiety. The potential mechanisms that could include the role of PA is in the regulation of stress responses via the hypothalamic-pituitary-adrenal (HPA) axis or glucocorticoids circulation. PA can help to reduce the symptoms of anxiety by promoting the manner of functioning of the brain regions which are particularly related to anxiety or stressrelated conditions. For example, the hippocampus is a region intrinsic for regulating the feedback of stress responses from the HPA. PA may also decrease the symptoms of anxiety through psychological mechanisms such as anxiety sensitivity [23].

It can thus be summated that all the parameters of the DASS-21 (Depression, Anxiety and Stress) have shown statistically significant differences in the pre and post intervention scores in both the control and experimental groups with $p$ values $<0.05$ in each domain. However, between the groups, significant difference could only be obtained in the domain of depression, whereas the domains of anxiety and stress have not shown any statistically significant difference.

\section{Conclusion}

According to the results obtained in the current study, mental imagery can be used as an effective adjunct to conventional aerobic exercises to reduce depression, anxiety and stress in the Leprosy affected population. Also, conventional aerobic exercises have a significant impact on the reduction of depression, anxiety and stress in this population. 


\section{Limitations}

The assessment of psychological parameters was based on the outcome measure, the DASS-21 which does not replace a face to face interview with a professional consultant for a clinical diagnosis of Depression, Anxiety or Stress disorders.

\section{Compliance with ethical standards}

\section{Acknowledgments}

We would like to thank Dr. Pradeep Chavan and the entire management of Dr. Bandorawalla Institute of Leprosy, Pune for their whole hearted support and cooperation. We would also like to thank our esteemed colleagues at Smt. Kashibai Navale college of Physiotherapy, Pune for their constant encouragement.

\section{Disclosure of conflict of interest}

The authors do not declare any conflict of interest.

\section{Statement of informed consent}

The study was conducted with due ethical considerations. Informed consent was obtained from all the participants in the study. There was no disclosure of identity of any of the participants.

\section{References}

[1] Lastória J, Abreu M. Leprosy: review of the epidemiological, clinical, and etiopathogenic aspects - Part 1. Anais Brasileiros de Dermatologia. 2014; 89(2):205-218.

[2] White C, Franco-Paredes C. Leprosy in the 21st Century. Clinical Microbiology Reviews. 2015;28(1):80-94.

[3] Leprosy (Hansen's disease) [Internet]. Who.int. 2021 [cited 16 December 2021]. Available from: https://www.who.int/news-room/fact-sheets/detail/leprosy

[4] Sengupta U. Elimination of leprosy in India: An analysis. Indian Journal of Dermatology, Venereology and Leprology. 2018;84(2):131.

[5] Kumar J, Verghese A. Psychiatric disturbances among leprosy patients. An epidemiological study. International Journal of Leprosy and other Mycobacterium Diseases. 1980; 48(4): 431-4.

[6] Behere P. Psychological reactions to leprosy. Leprosy India. 1981; 53(2): 266-72.

[7] Ramanathan U, Srivastav I, Ramu G. XII International Leprosy Congress Proceedings : New Delhi. Psychiatric morbidity in patients with leprosy - Review. 1984; 810-1.

[8] Shale M. A woman with leprosy is in double jeopardy. Leprosy Review. 2000;71(1).

[9] Jindal K, Singh G, Mohan V, Mahajan B. Psychiatric Morbidity Among Inmates of Leprosy Homes. Indian Journal of Psychological Medicine. 2013;35(4):335-340.

[10] Govindasamy K, Jacob I, Solomon R, Darlong J. Burden of depression and anxiety among leprosy affected and associated factors-A cross sectional study from India. PLOS Neglected Tropical Diseases. 2021;15(1):e0009030.

[11] Carek P, Laibstain S, Carek S. Exercise for the Treatment of Depression and Anxiety. The International Journal of Psychiatry in Medicine. 2011;41(1):15-28.

[12] Pearson J, Naselaris T, Holmes E, Kosslyn S. Mental Imagery: Functional Mechanisms and Clinical Applications. Trends in Cognitive Sciences. 2015;19(10):590-602.

[13] Reed T. Imagery in the Clinical Setting: A Tool for Healing. Nursing Clinics of North America. 2007;42(2):261277.

[14] Verma K, Gautam S. Psychiatric morbidity in displaced leprosy patients. Indian Journal of Leprosy. 1994; 66(3): 339-343.

[15] Coker A, Coker O, Sanni D. Psychometric properties of the 21-item Depression Anxiety Stress Scale (DASS-21). African Research Review. 2018;12(2):135. 
[16] Exercise for depression [Internet]. nhs.uk. 2021 [cited 16 December 2021]. Available from: https://www.nhs.uk/mental-health/self-help/guides-tools-and-activities/exercise-for-depression/

[17] Pile V, Williamson G, Saunders A, Holmes E, Lau J. Harnessing emotional mental imagery to reduce anxiety and depression in young people: an integrative review of progress and promise. The Lancet Psychiatry. 2021;8(9):836-852.

[18] Ignacio J, Dolmans D, Scherpbier A, Rethans J, Lopez V, Liaw S. Development, implementation, and evaluation of a mental rehearsal strategy to improve clinical performance and reduce stress: A mixed methods study. Nurse Education Today. 2016;37:27-32.

[19] Stephens R. Imagery. Clinical Nurse Specialist. 1993;7(4):170-174.

[20] Salmon P. Effects of physical exercise on anxiety, depression, and sensitivity to stress. Clinical Psychology Review. 2001;21(1):33-61.

[21] Schuch F, Vancampfort D, Richards J, Rosenbaum S, Ward P, Stubbs B. Exercise as a treatment for depression: A meta-analysis adjusting for publication bias. Journal of Psychiatric Research. 2016;77:42-51.

[22] Gujral S, Aizenstein H, Reynolds C, Butters M, Erickson K. Exercise effects on depression: Possible neural mechanisms. General Hospital Psychiatry. 2017;49:2-10.

[23] Kandola A, Vancampfort D, Herring M, Rebar A, Hallgren M, Firth J et al. Moving to Beat Anxiety: Epidemiology and Therapeutic Issues with Physical Activity for Anxiety. Current Psychiatry Reports. 2018;20(8). 